\title{
Le partenariat : Renouveau et défis dans les relations patronales-syndicales
}

\author{
Jean-François Tremblay, Ph. D. \\ Université du Québec en Outaouais
}

\section{PROBLÉMATIQUE}

Le terme « partenariat » se voit associé dans la littérature en relations industrielles à une nouvelle approche des relations de travail qui serait plus participative, plus coopérative et moins conflictuelle.

Au cours des dernières années, bon nombre d'expériences coopératives novatrices sont survenues entre employeurs et salariés. Plusieurs auteurs mentionnent que la coopération patronale-syndicale constitue la voie à privilégier afin de relever les défis de l'économie des années $2000^{1}$. Solution optimale afin de rencontrer à la fois les attentes de rentabilité des détenteurs du capital et les aspirations de démocratie industrielle des salariés, le partenariat entre employeurs et syndiqués devient dès lors pour certains le nouveau modèle de relations de travail à privilégier pour les années à venir. Apparu au début des années 1980 dans les dictionnaires de langue courante, le terme " partenariat » se voit associé dans la littérature en relations industrielles à une nouvelle approche des relations de travail qui serait plus participative, plus coopérative et moins conflictuelle. Émergeant d'un contexte idéologique prônant une transformation des relations antagonistes entre les acteurs sociaux vers une plus grande collaboration, la notion de partenariat est associée à de meilleures relations entre ces mêmes acteurs. La perspective d'un renouveau et d'une plus grande complicité dans les relations entre patrons et syndiqués semblent trouver preneur autant chez les praticiens que chez les académiciens.

Toutefois le concept de partenariat reste à ce jour un objet d'étude relativement embryonnaire et en cours d'élaboration conceptuelle. La désignation nominale du concept associé à diverses expériences de coopération souvent complexes et hétérogènes sème également la confusion sur la substance réelle du terme. C'est pourquoi il importe de s'y intéresser dans le cadre de la présente recherche et de proposer certaines pistes pouvant permettre une compréhension plus éclairante et structurante de cette notion complexe. Plus spécifiquement, le présent article propose 1) une description des différentes dimensions/composantes du partenariat patronalsyndical telles qu'identifiées dans la littérature scientifique et professionnelle; 2) la formulation pour fin de vérification d'une série de propositions de recherche concernant 8 composantes du construit de partenariat patronal-syndical regroupées en trois dimensions (nature, contenu et forme des ententes de partenariat); 3) l'étude de trois organisations ayant contracté des ententes de partenariat avec leurs salariés et finalement 4) une analyse transversale des attributs du partenariat observés dans les trois cas.

\section{DIMENSIONS/COMPOSANTES DU PROPOSITIONS DE RECHERCHE}

\subsection{Nature des ententes de partenariat}

\subsubsection{Statut des salariés}

On souligne la nécessité d'une redistribution du pouvoir au sein de l'organisation en faveur des employés et de leurs représentants désignés dans le cadre d'ententes de partenariat.

Il est mis en perspective dans la littérature que les ententes découlant d'un partenariat nécessitent une nouvelle reconnaissance du statut de chacun des acteurs et notamment celui de l'acteur syndical. On souligne la nécessité d'une redistribution du pouvoir au sein de l'organisation en faveur des employés et de leurs représentants désignés dans le cadre d'ententes de partenariat. 
Cette redistribution devrait amener une revalorisation du rôle et de la place des salariés (et du syndicat) au sein de l'organisation et doit donc être considérée comme un attribut important des ententes de partenariat. Cette nécessité de transformer l'alliance traditionnelle et de revaloriser le statut de l'acteur syndical est au cœur même du concept de partenariat. C'est pourquoi il serait donc possible de trouver dans les ententes de partenariat un ou des énoncés affirmant le nouveau rôle des salariés au sein de l'organisation et la nature de ce statut (position 1).

\subsection{2. Établissement d'un lien de confiance}

L'établissement d'un lien de confiance suffisamment « solide » entre les parties dans les expériences nécessitant la collaboration entre patrons et salariés est nécessaire afin de rompre la dynamique de méfiance qui caractérise bien souvent les relations traditionnelles entre les acteurs industriels. Dans la même veine, plusieurs auteurs mentionnent que si par le passé, la méfiance était souvent inhérente dans les rapports entre les acteurs, il devient impératif d'établir un lien de confiance solide entre les partenaires afin d'instituer un véritable partenariat entre eux. Le risque de voir l'une des parties poser un geste inattendu qui entraînerait un préjudice pour l'autre partie devrait se voir considérablement réduit lorsqu'employeurs et salariés évoluent en partenariat. Dès lors, la négociation de l'entente de partenariat par des agents qui cherchent à établir un lien de confiance entre eux n'auront pas besoin de l'intervention d'un tiers, pas plus qu'un recours à des moyens de pression tels que la grève et le lock-out afin $d$ 'arriver à une entente (position 2$)^{2}{ }^{2}$

\subsubsection{Partage de l'information}

\section{La signature d'une entente de partenariat serait conditionnelle à cette ouverture des parties concernant la transmission de l'information pertinente durant les négociations.}

Si traditionnellement il existe une asymétrie favorable à l'employeur dans la connaissance de l'information pertinente sur l'organisation, il importe qu'il existe un partage fluide de l'information dans le cadre des ententes de partenariat. L'entente de partenariat devrait comprendre des mécanismes d'accès à l'information plus substantiels. La signature d'une entente de partenariat serait conditionnelle à cette ouverture des parties concernant la transmission de l'information pertinente durant les négociations. Il nous importe donc de vérifier spécifiquement l'introduction de nouveaux processus qui visent à fournir aux salariés de l'information traditionnellement réservée à l'employeur. Les conventions collectives associées aux ententes de partenariat devraient donc comprendre des dispositions qui mentionnent que l'employeur met à la disposition des salariés les données opérationnelle, financière et stratégique de l'entreprise (position 3$)^{3}$.

\subsection{Contenu des ententes de partenariat}

\subsubsection{Nouvelles dispositions structurantes}

Le partenariat renvoie à une conception « gagnantgagnant » des relations patronales-syndicales. Pour les auteurs qui s'intéressent au partenariat patronalsyndical, il importe qu'il puisse y avoir dans l'entente de partenariat des gains de part et d'autre qui deviennent alors des gains structurants pour les acteurs. Plus de flexibilité pour l'employeur en échange d'une meilleure protection de l'emploi ou un partage de la plus-value pour les salariés semblent être les enjeux les plus importants proposés dans la littérature. Dans cette optique, l'entente de partenariat comporterait de nouvelles dispositions qui permettraient à l'employeur d'obtenir, par exemple, une plus grande flexibilité fonctionnelle ou salariale, alors que les employés auraient une plus grande part de la plus-value de l'entreprise, ou encore, une meilleure protection de l'emploi (position 4).

\subsubsection{Partage conjoint des responsabilités}

La littérature sur les expériences novatrices de coopération patronale-syndicale montre l'importance d'instituer des mécanismes de codécision afin de responsabiliser conjointement les parties et favoriser l'implication des salariés. C'est par l'instauration de mécanismes de codécision à tous les niveaux de l'organisation qu'il est possible de transformer les rapports sociaux au sein des organisations pour dépasser la simple logique de l'acceptation ou de la contestation des décisions de l'employeur en permettant aux salariés d'être partie prenante des 
différents processus organisationnels tout en favorisant des réflexes de conscientisation et d'imputabilité chez ces derniers. La codécision rééquilibre le pouvoir au sein de l'organisation créant ainsi une nouvelle dynamique qui tend à rapprocher les parties. La présence de mécanismes de codécision devrait pouvoir s'observer à partir des dispositions concernant les comités formellement reconnus dans le texte de la convention collective (position 5). Ces comités devraient également être paritaires afin d'actualiser un véritable partenariat (position 6) et doivent être présents aux différents niveaux de l'organisation milieu de travail, institutionnel et stratégique) (position 7).

\subsection{Forme des ententes de partenariat}

\subsubsection{Durée de l'entente}

Cherchant à établir des engagements communs sur une plus longue période afin d'atteindre les

\section{MÉTHODOLOGIE}

Pour les fins de cette étude exploratoire, nous avons étudié le cas de trois organisations de secteurs économiques distincts (municipal, pâtes et papiers et transformation alimentaire) ayant contracté des ententes de partenariat avec leurs employés. Par différentes méthodes de collecte des informations, soit l'analyse de contenu et l'entretien semi-directif, nous avons vérifié le bien-fondé de nos propositions de recherche. Afin de distinguer les particularités propres aux ententes de partenariat, nous avons effectué une comparaison entre la convention collective qui tient lieu d'entente de partenariat et celle qui fut signée lors de la précédente ronde de négociation avec une grille d'analyse conçue à cet effet. Cette comparaison nous permet de voir « émerger» des éléments particuliers ajoutés à la convention collective qui témoigneront de la nature et du contenu de l'entente de partenariat ${ }^{4}$. De plus, des entretiens semi-directifs ont été effectués avec les directeurs des relations de travail et les viceprésidents des syndicats des trois organisations étudiées. Des entrevues effectuées avec les représentants des parties avaient comme principaux objectifs de confirmer notre compréhension des modalités introduites dans les conventions collectives ainsi que d'approfondir objectifs fixés de part et d'autre, l'entente de partenariat est difficilement réalisable dans le cadre d'une convention collective traditionnelle. Considérant les difficultés inhérentes à l'implantation des mécanismes de coopération patronalesyndicale et leur fragilité, il importe que l'entente puisse inclure l'idée de pérennité afin de favoriser la concrétisation du partenariat. De plus, la stabilité organisationnelle qu'offre une entente de longue durée favorise l'innovation dans les règles de travail. Par ailleurs, on note que les innovations découlant de la coopération patronalesyndicale apparaissent et disparaissent souvent au gré des changements environnementaux, faisant en sorte de rendre aléatoire le succès des expériences de coopération. Nos attentes sont donc de retrouver des dispositions conventionnelles qui témoignent de la volonté des parties à s'engager sur une longue période dans le cadre de l'entente de partenariat (position 8 ).

certaines dimensions reliées au concept de partenariat comme la nature du lien de confiance avec les parties, le statut des salariés de même que les finalités recherchées par les parties dans le cadre du partenariat. Les entrevues, d'une durée approximative d'une heure trente minutes, furent complétées par des entretiens téléphoniques afin de valider l'analyse. Il est à noter que les intervenants rencontrés furent tous directement impliqués dans l'élaboration des ententes de partenariat.

Afin d'identifier les ententes patronalessyndicales qui renferment des modalités de partenariat, nous avons retenu celles qui ont déjà fait l'objet d'une analyse de contenu par des chercheurs en relations industrielles et qui ont été qualifiées, par ces mêmes chercheurs, d'ententes de partenariat ou de contrat social. Par la suite, notre souci fut de considérer des ententes qui avaient cours depuis un certain temps. La considération d'une entente signée trop récemment (moins d'un an) rendrait équivoque les constats fournis par les acteurs impliqués dans le partenariat patronal-syndical. 


\section{DESCRIPTION ET ANALYSE DES RÉSULTATS}

\subsection{Présentation du cas 1 : Une municipalité}

L'analyse comparative des conventions

collectives de cette municipalité nous a permis de constater la présence de

plusieurs dispositions qui convergent vers

les postulats théoriques du partenariat patronal-syndical.

Cette municipalité connaît depuis les dernières décennies de profonds changements qui ont modifié de manière importante son visage sociodémographique. La municipalité est confrontée à d'importants défis en matière de relations de travail. Les multiples conflits de travail survenus au cours des dernières années entre elle et ses salariés regroupés dans une section locale du Syndicat canadien de la fonction publique ont, par le passé, stéréotypé l'état des relations employeur-employés de cette organisation. Cependant, une volonté émanant des dirigeants politiques de même que de la part des dirigeants syndicaux a amené les parties à réformer leurs relations en tentant d'instituer entre elles un partenariat durable qui viserait à favoriser l'atteinte des objectifs particuliers de ces dernières. La convention collective tenant lieu d'entente de partenariat a une durée de 5 ans et comprend plusieurs nouvelles dispositions qui instituent les modalités et les finalités du partenariat. Il appert cependant que l'implantation de ce nouveau mode relationnel se heurte à une culture de confrontation et à un historique de relations de travail marqué par les conflits qui rendent difficiles la concrétisation des changements souhaités.

L'analyse comparative des conventions collectives de cette municipalité nous a permis de constater la présence de plusieurs dispositions qui convergent vers les postulats théoriques du partenariat patronalsyndical. Les dispositions de l'article 40, article qui a pour titre : « entente de partenariat », traitent spécifiquement et exclusivement du partenariat patronalsyndical. Plusieurs éléments qui y sont contenus reflètent parfaitement les prémisses et les principes du partenariat qui sont présentés dans la littérature, notamment la création de comités conjoints au niveau des lieux de travail et au niveau corporatif (art.40.04). Par ailleurs, l'article 39 sur la réorganisation du travail et la productivité constitue également une innovation de la dernière convention collective qui se doit d'être étroitement associée à l'entente de partenariat. Celui-ci précise diverses modalités qui permettent aux acteurs de mieux instituer les principes généraux du partenariat prévus à l'article 40. Il nous importe de mentionner que les dispositions des articles 39 et 40, sont des nouveautés inexistantes dans la précédente convention collective.

\subsection{Présentation du cas 2 : Une papetière}

Cette papetière québécoise est située en Mauricie. Les deux tiers de ses salariés sont des employés de production et de maintenance regroupés dans des unités locales affiliées à UNIFOR. Cette usine fut fermée en juin 1992 par ses anciens propriétaires et fut rachetée en 1993 par le Fonds de solidarité de la fédération des travailleurs du Québec qui en devint à ce moment le propriétaire unique. Conformément à sa politique d'investissement et de capitalisation, le Fonds se départit rapidement de ses actions au cours des années qui suivirent l'achat de l'entreprise pour les céder à un investisseur privé dont la philosophie de gestion concorde avec les principes directeurs du Fonds qui sont entre autres, la participation, l'implication et la formation des travailleurs de même que le partage des profits avec ces derniers.

L'analyse des données du cas $\mathbf{2}$ semble révéler l'existence d'un réel partenariat patronal-syndical mais dont l'essence peut difficilement se révéler à partir des seuls textes conventionnés. Conformément à la philosophie du Fonds, les salariés se sont retrouvés dans une organisation prônant la participation et l'implication intensives des salariés.

L'analyse des données du cas 2 semble révéler l'existence d'un réel partenariat patronal-syndical mais dont l'essence peut difficilement se révéler à partir des seuls textes conventionnés. Conformément à la philosophie du Fonds, les salariés se sont retrouvés dans une organisation prônant la participation et l'implication intensives des salariés. Ces mêmes salariés pouvaient même devenir coactionnaires de l'entreprise, via le Fonds, créant ainsi un lien avec l'employeur qui tend vers un partenariat d'affaire. Cette approche participative s'est 
également poursuivie lors du rachat de l'entreprise. On note toutefois que plusieurs modalités du partenariat ne sont pas conventionnées car les mécanismes de participation s'avèrent inhérents aux pratiques de gestion et ne font pas l'objet de dispositions dans la convention collective d'une durée de $4 \frac{1}{2}$ ans. À cet effet, les intervenants rencontrés nous ont toutefois souligné à maintes reprises qu'ils évoluaient en partenariat mais que la convention collective n'était pas nécessairement l'instrument approprié afin d'instituer ce mode relationnel.

\subsection{Présentation du cas 3 : Une entreprise de transformation alimentaire}

L'analyse du cas 3 permet d'inférer

l'existence d'un réel partenariat patronalsyndical. L'étude comparative des conventions collectives ainsi que les commentaires recueillis en entrevue nous laissent voir qu'il existe de nouvelles dispositions conventionnées et des pratiques de gestion qui favorisent une implication substantielle des salariés dans la gestion.

L'entreprise est une multinationale spécialisée dans la fabrication de chocolat de haute qualité et de produits dérivés du cacao. Possédant 40 usines réparties dans 25 pays et employant près de 7500 personnes, l'entreprise est un leader mondial dans l'industrie de la transformation du cacao. Animé par une volonté de consolider sa position de leader mondial, l'entreprise mise autant sur la recherche et le développement, l'innovation dans les méthodes de production du chocolat que dans une philosophie de relations de travail qui postule les bienfaits et la puissance du développement des compétences individuelles, l'implication des employés, l'ouverture dans les communications et la transmission de l'information, la créativité et l'innovation. Ce sont ces valeurs corporatives qui ont animé les dirigeants de l'usine québécoise située en Montérégie à signer un contrat social d'une durée de 6 ans avec leurs employés syndiqués affiliés à la CSN. La signature d'une telle entente était non seulement nécessaire afin de souscrire aux principes des dirigeants du groupe, mais également afin de réformer les relations de travail au sein de l'usine qui, depuis les années 1970, étaient, au dire des intervenants rencontrés, fort mauvaises ${ }^{5}$. Un investissement de 25 millions de dollars par la maison mère dans l'usine de St-Hyacinthe était conditionnel à la signature d'une convention collective de longue durée qui refléterait et opérationnaliserait cette philosophie de gestion. L'usine, dont la santé financière est excellente, souscrit aujourd'hui aux principes directeurs de gestion de la maison mère et forge des relations de travail hautement dynamiques et participatives avec ses employés.

L'analyse du cas 3 permet d'inférer l'existence d'un réel partenariat patronal-syndical. L'étude comparative des conventions collectives ainsi que les commentaires recueillis en entrevue nous laissent voir qu'il existe de nouvelles dispositions conventionnées et des pratiques de gestion qui favorisent une implication substantielle des salariés dans la gestion ainsi qu'une ouverture dans les communications et dans la transmission de l'information qui tendent vers les préceptes normatifs présentés dans la littérature sur le partenariat patronal-syndical. L'étude comparative des deux conventions collectives montre l'introduction de diverses clauses inexistantes dans l'entente précédente qui formalisent le partenariat entre les acteurs organisationnels. Se retrouvant sous le sous-titre « Contrat social et de travail de longue durée » en ouverture de la convention collective, cette section de l'entente regroupe les modalités de l'entente de partenariat. Les sous-sections du contrat social portent les titres de «Préambule » (A), « Négociation continue et processus de résolution de problèmes » $(\mathrm{B})$, " Comité des Relations Industrielles » (C), " Formation et de la qualification », « Réorganisation du travail ", "Réorganisation des mouvements de main-d'œuvre »(D) et « Communication » $(\mathrm{E})$. Ce sont sous ces thèmes que l'on retrouve la majorité des dispositions contractuelles négociées entre les parties dans le cadre de l'entente de longue durée.

\subsection{Analyse transversale des résultats}

4.4.1 Vérification des propositions se rapportant à la nature des ententes de partenariat

Tous les intervenants rencontrés ont affirmé l'importance du lien de confiance dans l'expérience de partenariat. Par ailleurs, nous avons également constaté que dans les trois cas, le partenariat patronal-syndical amène une évolution positive de la perception du lien de confiance. 
Nous avons trouvé un énoncé déclaratoire affirmant un nouveau statut pour les salariés (P1) dans deux des trois conventions collectives analysées : les conventions du cas 1 (art. 39.01 et 40.01) et du cas 3 (section A). Les termes « partenaire», «partenaires nécessaires », « partenaire premier » et « partenaires privilégiés » sont les substantifs et les qualificatifs consignés dans les conventions collectives afin de signifier le nouveau rôle des salariés. Bien qu'inexistante dans la convention collective du cas 2, cette dimension du nouveau statut des salariés s'est révélée d'une importance significative lors de l'analyse des entrevues. Le caractère implicite de ce changement dans le rôle des salariés n'amène pas, selon les intervenants rencontrés, la nécessité de le signifier dans la convention collective. Nous retenons donc que cette dimension du partenariat possède des assises empiriques bien qu'elle puisse être absente de la convention collective.

La proposition 2 sur le lien de confiance se voit vérifiée partiellement et amène une analyse équivoque des résultats. Il appert qu'aucun des trois cas n'a permis de confirmer pleinement notre proposition rattachée au lien de confiance. Dans le cas 1 et 3 , les ententes prirent plus de temps à être négociées et ont requis l'intervention d'un tiers. La présence de facilitateurs dans le processus de négociation fut requise dans les deux cas. Toutefois, les entrevues nous ont permis de constater l'importance cruciale de cette dimension du partenariat. Tous les intervenants rencontrés ont affirmé l'importance du lien de confiance dans l'expérience de partenariat. Par ailleurs, nous avons également constaté que dans les trois cas, le partenariat patronal-syndical amène une évolution positive de la perception du lien de confiance, bien que cette évolution puisse être lente, comme dans le cas 1 . Nous croyons que le manque de linéarité dans les résultats associés à la proposition 2 découle des limites de nos indicateurs de cette notion qui est, rappelons-le, complexe et dont l'opérationnalisation pose un défi. Nous retenons donc que l'établissement d'un lien de confiance est une dimension fondamentale du partenariat patronalsyndical, mais que son opérationnalisation, dans des études subséquentes, demande à être raffinée.

La possibilité que les salariés aient accès à plus d'information (P3) se voit vérifiée dans deux des trois cas avec cependant deux nuances importantes.
Dans les trois cas étudiés, ce ne sont pas les salariés pris individuellement qui ont reçu plus d'information mais bien le syndicat comme représentant institutionnel. Bien que les raisons varient d'un cas à l'autre, on remarque que l'accès direct pour les salariés à plus d'information pertinente généralement réservée à l'employeur sur la condition de l'entreprise n'était pas vraiment instauré lors de la négociation de l'entente de partenariat. Deuxièmement, seulement les cas 2 (art 5.01 et lettre d'entente no 3 ) et 3 (section A, B et E) nous amènent à conclure qu'il y a eu un réel partage de l'information avec les salariés ou avec leurs représentants. L'analyse du cas 1 démontre qu'il n'y a pas eu vraiment plus d'information transmise aux salariés. Par ailleurs, bien qu'il n'existe aucun mécanisme conventionné dans la convention collective du cas 2 , les salariés ont quand même accès à l'information de nature stratégique via leur représentant au conseil d'administration. Toutefois, l'analyse des deux autres cas montrent clairement que le partage de l'information stratégique n'est pas institué entre les acteurs même s'il existe des mécanismes conventionnés à cet effet. Nous retenons donc que le partenariat patronal-syndical amène la création de mécanismes novateurs de transmission de l'information permettant aux salariés de mieux apprécier la condition de leur organisation.

\subsubsection{Vérification des propositions se rapportant au contenu des ententes de partenariat}

La proposition 4 se voit spécifiquement vérifiée dans deux des trois cas. À cet effet, on remarque à la section F) sur les mouvements de main-d'œuvre de la convention collective du cas 3 , la possibilité pour l'employeur de recourir à une équipe de travail dite « volante " pour les surcroîts de travail et un plancher d'emploi pour les salariés. Toutefois, il appert que l'analyse du cas 1 (art. 1.02; 5.10; 39.01; 40.01) nous montre qu'en nombre absolu, les salariés ont vu leur plancher d'emploi diminué alors que dans le cas 2 (art. 8 et lettre d'entente no 21), la protection de l'emploi des salariés passe essentiellement par une restriction dans le droit à la soustraitance pour l'employeur. Mentionnons cependant qu'à aucun moment, les intervenants syndicaux rencontrés en entrevue n'ont mentionné qu'ils sentaient que les dispositions négociées à ce sujet entrainaient une baisse dans la protection des emplois ${ }^{6}$. Nous retenons donc que plus de flexibilité fonctionnelle pour l'employeur en échange d'un 
plancher d'emploi ou d'une restriction dans le recours à la sous-traitance semble être le compromis « gagnant-gagnant » associé aux trois ententes de partenariat étudiées. Ces observations, de même que les commentaires recueillis lors des entrevues, nous amènent à retenir que les ententes de partenariat doivent effectivement avoir des dispositions « accommodantes » pour chacun des partenaires bien qu'il soit difficile d'apprécier jusqu'à quel point ces innovations conventionnées constituent une réelle amélioration (ou une détérioration) de la condition des parties.

L’analyse agrégée des dispositions
conventionnées nous montre que les ententes
de partenariat amènent dans les trois cas la
création de mécanismes de concertation
patronale-syndicale qui prennent
principalement la forme de comités
paritaires « décisionnels ».

Les résultats des analyses associés aux propositions 5,6 et 7 nous ont permis de soutenir partiellement les propositions. L'analyse agrégée des dispositions conventionnées nous montre que les ententes de partenariat amènent dans les trois cas la création de mécanismes de concertation patronalesyndicale qui prennent principalement la forme de comités paritaires « décisionnels ${ }^{7}$ dont les mandats ont essentiellement pour but de discuter d'enjeux concernant les changements organisationnels, les changements technologiques et les changements dans les processus de relations de travail. Par ailleurs, l'analyse détaillée des trois cas nous permet de constater que les objets du partenariat sont vastes et multiples mais qu'ils concernent rarement des enjeux d'ordre stratégique. Par exemple, l'entente propre au cas 1 montre que les dispositions des articles $39.01,39.05$ e) et 39.07 f) indiquent que les changements de nature technologique sont au cœur de l'entente. De la même façon, les dispositions conventionnelles des articles 39.01, $39.07 \mathrm{a}$ ), $39.07 \mathrm{c}$ ), $39.07 \mathrm{~g}$ ) et 40.03 e) montrent l'ensemble des sujets se rapportant aux changements. La sous-traitance, l'amélioration de la prestation de travail, l'étude des nouvelles méthodes de travail et la recherche d'une organisation du travail qui permet une meilleure productivité sont quelques exemples des sujets de discussion qui découlent de l'entente de partenariat.
Finalement, les dispositions des articles 39.08, $40.01,40.02,40.03 \mathrm{~b}$ ), $40.03 \mathrm{f}$ ), $40.03 \mathrm{~g}$ ) et $40.04 \mathrm{~b}$ ) mettent en lumière de nouveaux mécanismes qui transforment les mécanismes de régulation des relations industrielles au sein de l'organisation. La renonciation à la procédure de grief concernant les dispositions de l'article sur la réorganisation du travail et la productivité, l'instauration des comités de partenariat, la nécessité de la paix industrielle sur une longue période, la médiation préventive et les nouveaux mécanismes de discussion patronalesyndicale sont autant d'innovations qui découlent de l'entente et qui transforment le procès de relation de travail. De la même façon, on retrouve dans la convention collective du cas 3 (sections A, B, C, D et $\mathrm{E}$ ) des mécanismes de discussion permettant aux acteurs de se pencher sur des enjeux tels que la mise en place des changements requis en regard de la nouvelle technologie, l'analyse des postes, l'implantation de programmes d'amélioration continue, l'élaboration de programmes de formation, l'opportunité de recourir à la sous-traitance, la réaffectation des salariés mis à pied, la création d'équipes semiautonomes, la réorganisation du travail, les processus de négociation continue et de résolution de problèmes et l'introduction d'un mécanisme d'arbitrage de l'offre finale. Dans le cas 2, les salariés semblent cependant peu préoccupés à s'impliquer au niveau stratégique de l'organisation. Une des raisons pouvant être avancée, et qui découle de l'analyse des entrevues, est à l'effet que les salariés font confiance aux dirigeants de l'entreprise. Ils sont toutefois impliqués au niveau des lieux de travail avec le comité sur l'automation (art. 7.01) et celui sur la description et la classification des nouveaux emplois (lettre d'entente no 22).

La constatation du peu de présence ouvrière au niveau stratégique va à l'encontre de la littérature sur le partenariat patronal-syndical qui nous laissait entrevoir la nécessaire participation des salariés au niveau stratégique, de même que des discussions sur des enjeux dits corporatifs. Il est donc nécessaire, à la lumière des constats empiriques de cette étude, de nuancer les considérations théoriques retenues jusqu'à présent qui proposaient explicitement la création de mécanismes codécisifs, la participation des salariés aux trois niveaux de l'activité industrielle et un apport des salariés dans les discussions reliées aux enjeux stratégiques. Dans les trois cas étudiés, ce sont les niveaux des lieux de 
travail et institutionnel qui laisse place au partenariat patronal-syndical alors que l'implication au niveau stratégique est beaucoup plus « timide».

\subsubsection{Vérification de la proposition se rapportant à la forme des ententes de partenariat}

Les conventions collectives du cas 1 (art. 40.04 b) et 3 (section A) renferment toutes deux une modalité qui exprime clairement la nécessaire longévité de l'entente (P8). Qui plus est, même s'il n'existe aucune disposition à cet effet dans la convention collective du cas 2 , les intervenants rencontrés nous ont signifié qu'ils souhaitaient pouvoir s'entendre sur la signature d'une convention collective de longue durée lors du renouvellement de la prochaine convention. On peut donc retenir que les ententes de partenariat sont associées à des conventions de longue durée et qu'il existe chez les partenaires une propension à s'engager sur une longue période.

\subsection{Synthèse de l'analyse}

Nous présentons au tableau 2, la synthèse de l'analyse proposée précédemment. Reprenant les trois dimensions du construit et les propositions associées à chacune de ses composantes, nous proposons ici les résultats de l'analyse pour chacun des trois cas et cela, en regard de l'analyse des conventions collectives et des entrevues. Le tableau synthèse permet de constater que chacune des propositions de recherche, à l'exception de la septième, se voit soutenue par l'analyse des conventions collectives dans au moins deux des trois cas. Comme il n'existe pas de critère de décision précis pour ce type d'étude exploratoire, nous considérerons nos propositions de recherche comme étant soutenues lorsqu'elles se vérifient dans au moins deux des trois cas. Par ailleurs, la synthèse de l'analyse des résultats d'entrevues permet quant à elle de mettre en relief l'écart entre la règle conventionnée et les comportements tels que perçus par les intervenants.

\section{Tableau 1 - Tableau synthèse de l'évaluation du partenariat}

X: Proposition soutenue; X-:Jugé important mais non conventionné; O: Proposition infirmée; $\mathrm{O}+$ : Conventionnée mais non-appliquée.

\begin{tabular}{|c|c|c|c|c|c|c|c|}
\hline & \multicolumn{6}{|c|}{ CAS ET OUTILS D'ÉVALUATION } \\
\hline & & \multicolumn{2}{|c|}{1} & \multicolumn{2}{|c|}{2} & \multicolumn{2}{|r|}{3} \\
\hline DIMENSION & PROPOSITION & $\begin{array}{l}\text { ANALYSE } \\
\text { C.C. }\end{array}$ & ENTREVUE & $\begin{array}{l}\text { ANALYSE } \\
\text { C.C. }\end{array}$ & ENTREVUE & $\begin{array}{l}\text { ANALYSE } \\
\text { C.C. }\end{array}$ & ENTREVUE \\
\hline NATURE & \begin{tabular}{|c}
$\begin{array}{c}\text { Statut du salariés } \\
(\mathrm{P} 1)\end{array}$ \\
\end{tabular} & $\mathrm{X}$ & $\mathrm{O}+$ & $\mathrm{O}$ & $\mathrm{X}-$ & $\mathrm{X}$ & $\mathrm{X}$ \\
\hline DES & $\begin{array}{l}\text { Confiance: } \\
\text { (P2) }\end{array}$ & $\begin{array}{c}\text { ne } \\
\text { s'applique } \\
\text { pas }\end{array}$ & $\mathrm{O}$ & $\begin{array}{c}\text { ne s'applique } \\
\text { pas }\end{array}$ & $\mathrm{X}$ & $\begin{array}{c}\text { ne s'applique } \\
\text { pas }\end{array}$ & $\mathrm{X}$ \\
\hline ENTENTES & $\begin{array}{c}\text { Partage de } \\
\text { l'information } \\
\text { (P3) }\end{array}$ & $\mathrm{X}$ & $\mathrm{O}+$ & $\mathrm{X}$ & $\mathrm{X}$ & $\mathrm{X}$ & $\mathrm{O}+$ \\
\hline CONTENU & $\begin{array}{c}\text { Dispositions } \\
\text { structurantes } \\
\text { (P4) }\end{array}$ & $\mathrm{X}$ & $\mathrm{O}+$ & $\mathrm{X}$ & $X$ & $\mathrm{X}$ & $\mathrm{X}$ \\
\hline DES & $\begin{array}{c}\text { Comités } \\
\text { conjoints } \\
\text { (P5) }\end{array}$ & $\mathrm{X}$ & $\mathrm{X}$ & $\mathrm{X}$ & $\mathrm{X}$ & $\mathrm{X}$ & $\mathrm{X}$ \\
\hline & $\begin{array}{l}\text { Mécanismes de } \\
\text { codécisions } \\
\text { (P6) }\end{array}$ & $\mathrm{X}$ & $\mathrm{O}+$ & $\mathrm{X}$ & $\mathrm{X}$ & $\mathrm{X}$ & $\mathrm{X}$ \\
\hline ENTENTES & $\begin{array}{c}\text { Niveau } \\
\text { d'implication } \\
\text { (P7) }\end{array}$ & $\mathrm{O}$ & $\mathrm{O}$ & $\mathrm{O}$ & $\mathrm{X}-$ & $\mathrm{O}$ & $\mathrm{O}$ \\
\hline $\begin{array}{l}\text { FORMES } \\
\text { DES } \\
\text { ENTENTES }\end{array}$ & $\begin{array}{l}\text { Contrat de } \\
\text { longue durée } \\
\text { (P8) }\end{array}$ & $\mathrm{X}$ & $\mathrm{X}$ & $\mathrm{O}$ & $X-$ & $\mathrm{X}$ & $\mathrm{X}$ \\
\hline
\end{tabular}

Organisations \& territoires (2015) 


\section{CONCLUSION}

Nos analyses empiriques tendent également à démontrer que le partenariat patronalsyndical est un « work in progress " dont la forme optimale reste encore indéterminée.

Il appert que les composantes identifiées dans notre étude aient des assises empiriques dans les ententes de partenariat des trois cas étudiés. Il nous fut possible de retrouver de manière formelle dans les conventions collectives les composantes associées au construit. Toutefois, nos analyses tendent à démontrer que les salariés ou leur syndicat sont peu impliqués au niveau stratégique de l'organisation et dans les discussions qui relèvent de ce niveau. Dès lors, nous croyons que le construit proposé dans le cadre de cette recherche s'avère un référentiel théorique pertinent afin de qualifier une entente de partenariat. Nos analyses empiriques tendent également à démontrer que le partenariat patronalsyndical est un «work in progress » dont la forme optimale reste encore indéterminée. Nous avons cependant observé un réel élargissement des enjeux de la négociation entre employeur et salariés, de même qu'une conscientisation des acteurs face à leurs besoins communs et respectifs. Par ailleurs, il nous est possible de formuler une définition du partenariat patronal-syndical qui tient compte à la fois des considérations théoriques et empiriques révélées dans cette étude :

Le partenariat patronal-syndical est l'alliance formelle des acteurs industriels dans le cadre d'une entente bilatérale à moyen ou à long terme visant la réalisation d'objectifs communs dont les résultats escomptés pourront bénéficier aux partenaires tout en revalorisant le statut des salariés au sein de l'organisation. Le partenariat patronal-syndical est notamment caractérisé par une relation de confiance accrue entre les parties, une participation des salariés à tous les niveaux de l'organisation, un élargissement des enjeux de la négociation et un partage plus équilibré de certains pouvoirs et de certaines responsabilités traditionnellement conférés à l'employeur.
$\mathrm{Au}$ terme de cet article, nous croyons apporter une contribution significative à l'étude du partenariat patronal-syndical malgré les limites inhérentes associées aux études exploratoires et aux études de cas. Dans un premier temps, nous avons été à même de conceptualiser sous forme de construit théorique la notion de partenariat patronal-syndical. Alors que plusieurs auteurs réfèrent au partenariat patronal-syndical, aucune étude n'a cherché jusqu'à ce jour à décrire le concept de manière précise. La considération du concept et de ses attributs comme un objet d'étude en soi peut donc se révéler comme étant un élément théorique pertinent dans notre compréhension de la nature et de l'évolution des rapports entre employeurs et salariés. De plus, au niveau méthodologique, notre étude suggère qu'il peut s'avérer problématique de ne considérer que le contenu des conventions collectives afin de conclure ou non à l'existence d'un réel partenariat patronal-syndical au sein d'une organisation. La seule considération de la règle écrite peut nous amener à sous-estimer l'ampleur du partenariat (comme dans le cas 2) ou à le surestimer (comme dans le cas 3).

Les intervenants rencontrés nous ont signifié que le partenariat était un but à atteindre; ils le considèrent plutôt comme un moyen d'atteindre des objectifs « traditionnels » comme la croissance et la rentabilité de l'organisation pour l'employeur et l'amélioration des conditions de travail et d'emploi pour les salariés.

Finalement, la description de trois cas aussi distincts permet d'apprécier la richesse et l'hétérogénéité des ententes de partenariat patronalsyndical, rejetant par le fait même le caractère univoque du concept. L'analyse de contenu des entrevues nous permet de constater que les intervenants jugent le partenariat patronalsyndical comme étant un outil malléable, un moyen pour arriver à une fin qui appartient à chacun des acteurs. Dans aucun des trois cas, les intervenants rencontrés nous ont signifié que le 
partenariat était un but à atteindre; ils le considèrent plutôt comme un moyen d'atteindre des objectifs " traditionnels » comme la croissance et la rentabilité de l'organisation pour l'employeur et l'amélioration des conditions de travail et d'emploi pour les salariés

\section{BIBLIOGRAPHIE ET NOTES}

${ }^{1}$ Kochan, T.A., P.A. Adler, R.B. McKersie, A.E. Eaton, P. Segal et P. Gerhat. (2008). "The Potential an Precariousness of Partnership: The Case of the Kaiser Permanente Labor Management Partnership". Industrial Relations. Vol. 47, no 1 , p. 36-65.

${ }^{2}$ Bien sûr, cet indicateur s'avère imparfait afin de qualifier la confiance entre les parties. Toutefois, les entretiens effectués avec les représentants des parties devraient nous permettre de mieux circonscrire l'état du lien de confiance entre elles. La nature des données disponibles ne nous permet pas une meilleure opérationnalisation de cette composante.

${ }^{3}$ Mentionnons cependant qu'on pourrait observer une absence de telles spécifications dans la convention collective bien qu'effectivement les salariés aient accès à ces informations. L'information serait alors disponible indirectement via la présence de représentants syndicaux au conseil d'administration de l'entreprise ou par d'autres mécanismes.

${ }^{4}$ Nous avons également analysé des données colligées par les services de ressources humaines des différentes organisations sur le processus ayant conduit à ces ententes: à savoir, l'étape du règlement et la nature de l'intervention d'un tiers (si elle a eu lieu) dans le processus de négociation.

${ }^{5}$ Épée de Damoclès s'il en est une, la fermeture de l'usine italienne du Groupe pour cause de mauvaises relations de travail a certes influé, aux dires des intervenants rencontrés, sur leur propension à réformer leurs relations de travail pour les amener sur la voie de la coopération afin de solutionner les maux chroniques de relations de travail omniprésents depuis 30 ans. En fait, il appert que les multiples conflits qui caractérisaient l'usine québécoise étaient très mal perçus par les hauts dirigeants de la maison mère et que ces derniers ont identifié comme étant le principal défi, la réforme de leurs relations de travail qui amènerait les parties à «éradiquer» les conflits de travail et instaurer un partenariat entre elles.

${ }^{6}$ Notons qu'au moment des entrevues, nous avons explicitement fait référence aux clauses qui, selon nous, donnaient plus de flexibilité à l'employeur et celles procurant une meilleure protection des emplois. À la lumière de notre analyse, les intervenants auraient pu faire des nuances à ce sujet, mais aucun d'entre eux ne l'a fait. Ces derniers ont simplement corroboré notre analyse.
${ }^{7}$ Dans les trois cas, les décisions des comités se doivent d'être sanctionnées par les instances compétentes. Les entrevues révèlent cependant que les décisions de ces comités sont rarement renversées. 